\title{
HISTORIA Y TRANFORMACIÓN DE LA TELEVISIÓN DE PAGO EN ESPAÑA. UN RECORRIDO TECNOLÓGICO DESDE EL VÍDEO COMUNITARIO HASTA EL VÍDEO ONLINE.
}

\author{
María Luisa García-Guardia1: Universidad Complutense de Madrid, España \\ mgg@interacting.es
}

Óscar Estupiñán-Estupiñán: Universidad Complutense de Madrid, España oje791207@gmail.com

\section{RESUMEN}

La industria de televisión de pago en España, desde su aparición, a finales de la década de los 70, se encuentra en constante transformación. Tres factores han permitido esta continua evolución: a. La digitalización de la televisión, b. la legislación sobre el uso y competencias de estos nuevos servicios, c. la posibilidad de nuevas formas de negocio que permitieron que la utilización de Internet, y en especial, la utilización de la banda ancha creara un nuevo formato de consumo audiovisual: el vídeo online. El vídeo online se convierte hoy por hoy en un nuevo paradigma de negocio. Su consumo es el que más ha crecido en el país, a la vez, que se constituye en una amenaza para un sector, que como el de Televisión de Pago necesita, nuevamente, de redefiniciones en sus ofertas de contenidos y consumos, haciendo que dicha amenaza se convierta en una oportunidad de negocio. Esta investigación realiza un recorrido histórico por la transformación y evolución de la Televisión de pago en España hasta llegar al auge del vídeo online teniendo presente cómo han incidido las tecnologías, las legislaciones y los conglomerados económicos.

PALABRAS CLAVE: Televisión de pago - Vídeo online - Televisión de pago por cable - Satélite - ADSL

\footnotetext{
${ }^{1}$ Autor correspondiente María Luisa García-Guardia: Profesora en la Facultad de Ciencias de la Información. Universidad Complutense de Madrid, España.

Correo: $\underline{m g g @ i n t e r a c t i n g . e s ~}$
} 


\title{
HISTORY AND TRANSFORMATION OF PAY TELEVISION IN SPAIN. A TECHNOLOGIAL TOUR FROM TO VIDEO COMMUNITY TO VIDEO ONLINE.
}

\begin{abstract}
The pay television industry in Spain since its appearance in the late 70's is in constant transformation. Three factors have allowed this continual evolution: a. the digitization of television, $b$. legislation on the use and powers of these new services, $c$. the possibility of new forms of business that allowed the use of Internet and especially the use of broadband will create a new form of audiovisual consumption: the video online. Online video today becomes a new business paradigm. Its consumption is the fastest growing in the country at a time, which constitutes a threat to a sector, such as pay TV needs again redefinitions in its content offerings and consumption, causing it to threaten to become a business opportunity. This research takes a historical transformation and evolution of Pay TV in Spain until the rise of online video bearing in mind how they have impacted the technologies, laws and economic conglomerates.
\end{abstract}

KEY WORDS: Pay TV - Online Video - Pay TV Via Cable - Satellite - ADSL

\section{INTRODUCCIÓN}

El reto que representa el aumento de la oferta y del consumo del vídeo online en el actual modelo de negocios de la industria de televisión de pago en España, y las razones que han motivado a esta industria ha convertir esta amenaza en una oportunidad es el eje central de esta investigación.

El artículo analiza la transformación y evolución de esta industria en España desde su nacimiento hasta nuestros días, realizando un recorrido por tres aspectos centrales: la tecnología (digitalización de la imagen), la legislación (Leyes y Reales Decretos) y la creación de nuevos conglomerados económicos, que a su vez, permitieron que la televisión de pago se transformará en lo que conocemos hoy en día.

La investigación realiza un recorrido histórico sobre cómo los avances tecnológicos y los cambios en los hábitos de consumo (que son el resultado del aumento del consumo de Internet y de la penetración de la banda ancha), están delineando un nuevo panorama audiovisual, al que la industria de televisión de pago se debe y se está adaptando, si desea continuar, según las respuestas de nuestros sujetos de estudio, con su crecimiento económico, preservando así a sus abonados. 


\section{METODOLOGÍA}

\subsection{Objetivos}

a) Analizar y estudiar la transformación y evolución de la televisión de pago en España hasta la aparición del vídeo online y su penetración en la banda ancha de Internet.

b) Seleccionar cuáles son los factores que han influido en la transformación y evolución de la televisión de pago en España hasta la aparición del vídeo online.

\subsection{Herramientas metodológicas}

Para la recolección y análisis de los datos se utilizarán herramientas de tipo cualitativo y cuantitativo, en un proceso de retroalimentación constante que permitirá contrastar los objetivos de estudio, el estado de la cuestión y la hipótesis planteada. Se han seleccionado para ambos análisis dos herramientas metodológicas a saber:

\subsubsection{Herramienta de análisis cualitativo:}

Entrevista en profundidad: Se realizarán cuestionarios en forma de entrevistas en profundidad que contienen las siguientes unidades temáticas:

1. La televisión de pago en España

- Historia

- Legislación

- Conglomerado económico

2. Televisión de pago por cable, satélite y ADSL

- Historia

- Legislación

- Conglomerado económico

3. Vídeo online

- Características

- Modalidades

- Soporte de visionado

\subsubsection{Herramienta de análisis cuantitativo}

Análisis de contenido: Una vez finalizadas las entrevistas y su posterior trascripción se procederá a una codificación y análisis de los contenidos, al igual que se segmentarán, dichas entrevistas, según las unidades temáticas seleccionadas, buscando una relación entre las mismas.

El software que se utilizará permitirá la codificación tanto de las unidades semánticas 
como de los párrafos de las frases en los cuales intervienen los entrevistados.

\subsection{Selección de la muestra}

a) 2 profesionales especializados en la industria de Televisión de pago en España

b) 2 profesionales especializados en la industria de Internet en España, con énfasis en la transmisión y comercialización de contenidos audiovisuales a través de la red y del vídeo online.

\subsection{Características de la selección de la muestra}

a) Los profesionales especializados tienen más de 10 años de experiencia en el sector, lo que permite un análisis más detallado sobre la transformación que ha sufrido la industria de contenidos audiovisuales en España.

b) Los profesionales tienen experiencia en los procesos de producción y distribución de contenidos audiovisuales, permitiendo conocer de primera mano todos los procesos en los que se apoyan los contenidos en la industria de Televisión de pago para ser comercializados

Finalizadas las entrevistas, se tendrá en cuenta el método de Krippendorff ${ }^{2}$ y que se resume a continuación:

Tabla 1. Método Krippendorff

\begin{tabular}{|c|c|}
\hline Estrategia metodológica & Análisis de contenido \\
\hline Software de apoyo & QSR Nvivo 8.0 \\
\hline Procedimientos & $\begin{array}{c}\frac{\text { Muestreo: 4 entrevistas en profundidad }}{\text { con personas representativas }} \\
\text { Registro: Trascripción del audio, } \\
\text { preparación del software de análisis y } \\
\text { clasificación semántica }\end{array}$ \\
$\begin{array}{c}\text { Reducción de los datos: Verificación de } \\
\text { las respuestas a las preguntas de } \\
\text { investigación }\end{array}$ \\
\hline
\end{tabular}

Fuente: Elaboración propia.

\footnotetext{
2 Krippendorff, Klaus (1990). Metodología de Análisis de contenido: Teoría y práctica, Barcelona: Paidós
} Comunicación. 


\section{ANÁLISIS Y DISCUSIÓN}

\subsection{El nacimiento de la televisión de pago en España. Transformación y evolución}

\subsubsection{Primeras experiencias de Televisión por Cable en los años 70-80}

La primera experiencia de televisión por cable en España, y por consiguiente, de TV de pago, surge en 1972 como resultado de una iniciativa de la Dirección General de Radiodifusión y Televisión y de la compañía Telefónica. El proyecto consistía en instalar una red de cable coaxial en Madrid y en Barcelona, dando servicio a 30.000 hogares aproximadamente. Las redes se instalaron, pero el servicio nunca entró en funcionamiento.

A principios de los 80, entraron en funcionamiento las primeras redes de cable, principalmente, en el sur y en el levante del país. Las redes de cable siguieron el modelo de los sistemas de antena comunitaria (CATV - Community Antenna Television), implantados en Estados Unidos. Pero a diferencia del sistema americano, en España no se instalaron inicialmente las MATV (Master Antenna Television) para difundir las señales de televisión tradicional, dada la limitada oferta televisiva con la que contaba el país en esta década, sino que se utilizaba el sistema de antena colectiva de televisión para difundir la señal de un reproductor de vídeo, a cambio de un canon que se pagaba al prestador/administrador del servicio. A este servicio se lo conoció con el nombre de "Vídeo Comunitario", y cuyos derechos de "autor" para la reproducción y difusión de estos vídeos, generalmente no se pagaban.

El sistema de "Vídeos Comunitarios" se extendió por la geografía nacional, creándose en 1988 la Asociación de Vídeos Comunitarios (AVIDECO), que se convirtió en la defensora de los derechos de estos sistemas de cable ante los tribunales, dada su precaria situación de legalidad.

En 1989 se creó la Asociación Española de Servicios de Distribución por Cable (AESDICA), coincidiendo con la aparición de las televisiones privadas generalistas. Entre los objetivos de la Asociación se encontraba presionar a la Administración Central para que regulase su actividad. Para esta fecha existían los primeros sistemas de cable comunitario o CATV's que emitían, además de los canales generalistas españoles, canales extranjeros, la mayoría procedentes de satélites e incluso, añadían un canal de producción propia, de carácter local, para hacer más atractivo el servicio.

Finalmente en 1995, se promulgó la Ley $42 / 1995^{3}$ de las Telecomunicaciones por Cable, que definía el servicio de telecomunicaciones por cable como público y de titularidad estatal, determinando que la licencia de su prestación se obtendría mediante concurso público. Entre los objetivos de la Ley se encontraban:

\footnotetext{
${ }^{3}$ Boletín Oficial del Estado. Ley 42/ 1995, de 22 de diciembre, de las Telecomunicaciones por cable. BOE, número 0306, 23 de diciembre de 1995.
} 
a. Dotar al servicio de una ordenación inicial que lo sacase del marco de la alegalidad.

b. Sentar un marco de referencia para el desarrollo de la industria del cable en España.

A partir de la aprobación de la Ley 42/1995 de telecomunicaciones por cable (LTC), se convocaron concursos y se otorgaron licencias para explotar comercialmente sistemas de cable en las distintas demarcaciones territoriales. Posteriormente, se publicó la Ley General de Telecomunicaciones de 19984, que derogaba a la anterior y en la que los operadores de cable se enmarcaban bajo un doble régimen regulatorio: a. El que regula el ámbito de las telecomunicaciones. b. El que regula el ámbito de la difusión televisiva. Este doble régimen regulatorio mantuvo una situación singular en la que la liberalización de las telecomunicaciones por cable se producía en paralelo al mantenimiento de las restricciones para prestar el servicio de difusión de televisión. Expansión de los sistemas de Cable: La década de los noventa.

Los concursos que fueron sucediéndose para la obtención de licencias para explotar comercialmente redes de cable en las distintas demarcaciones dieron como resultado un mapa empresarial del cable a finales de los noventa, concentrado bajo el dominio de dos operadores de telecomunicaciones: Retevisión y Cableuropa, que participaban de manera directa en el capital de los operadores de cable, o bien a través de socios accionistas.

Retevisión contaba, mayoritariamente, con la participación de las eléctricas Unión Fenosa y Endesa, y el operador de telecomunicaciones Telecom Italia. Los socios de Retevisión crearon distintas redes de cable asociadas, y también las empresas Amena (telefonía móvil), Onda Digital (televisión digital terrestre), EresMás (acceso a Internet). Sin embargo, en junio del 2000 nace AUNA, que agrupa las participaciones de Endesa, Telecom Italia y Unión Fenosa en: Retevisión, Amena, EresMás, Quiero TV, Hispasat y los operadores de cable: Menta (Cataluña), Madritel (Madrid), Supercable (Andalucía), Cabletelca (Canarias), R (Galicia) y Able (Aragón).

Por otro lado, Cableuropa contó con la participación, mayoritaria, de las empresas Ferrovial, Multitel Cable, Banco Santander Central Hispano, y el consorcio americano Spaincom. No obstante, la marca comercial de los servicios de cable ofrecidos por los operadores que contaban con participación en Cableuropa, fue ONO.

Los sistemas de cable ofrecen a los socios de referencia de Retevisión y Cableuropa, la posibilidad de ampliar sus fronteras de negocio, hacia la televisión, provisión de acceso a Internet y servicios interactivos, y de esta manera competir con la oferta de servicios del Grupo Telefónica, operador dominante en España del sector de las telecomunicaciones.

La década de los noventa se caracterizó por el lanzamiento y expansión de los

\footnotetext{
${ }^{4}$ Boletín Oficial del Estado. Ley 11/1998, de 24 de abril, General de las Telecomunicaciones. BOE, número 99, 25 de abril de 1998.
} 
sistemas de cable, a partir de las licitaciones que se fueron adjudicando, y por otro lado se conformaron los grupos económicos que concentraban gran parte de estas operaciones. En un informe publicado en el año 2000 por la Comisión del Mercado de las Telecomunicaciones (CMT), se afirmaba que: "1999 ha sido el año en el que, en términos generales el cable ha comenzado su andadura propiamente dicha". ${ }^{5}$

Desde el punto de vista jurídico, la Ley de las Telecomunicaciones por Cable fue derogada en su totalidad por la Ley General de Telecomunicaciones 32/20036, de noviembre de 2003, que liberalizaba la prestación del servicio de difusión de televisión por cable, y por lo tanto dejaba de ser servicio público. Aunque por una disposición de esta Ley se aplazaba la entrada en vigor de esta liberalización hasta el 31 de diciembre de 2009, ésta se produjo gracias al Real Decreto 920/2006, de 20067, por el que se aprueba el Reglamento General de Prestación del Servicio de difusión de radio y televisión por Cable. Una de las disposiciones de este Real Decreto destacaba la obligación a los concesionarios del servicio de televisión por cable a incluir en su oferta, hasta el cese definitivo de las emisiones de televisión con tecnología analógica, los canales nacionales terrestres y los públicos autonómicos.

\subsection{La televisión digital de pago por cable en España}

Desde el punto de vista tecnológico, la TV de pago se desarrolló sobre la tecnología que se utilizaba para la televisión tradicional, es decir, la analógica. La digitalización de esta industria marca una nueva fase de evolución que viene a catalizar los procesos, antes comenzados, y a abrir nuevas posibilidades en la producción y distribución de los contenidos y canales de pago.

El impacto que genera la digitalización en la Televisión de pago, en este caso el español, abre definitivamente las puertas a la convergencia efectiva entre la televisión, la informática y las telecomunicaciones, transformando el sector, en una dimensión macro, en cuanto a la capacidad de producción, emisión y consumo de medios audiovisuales.

... en España, una implicación directa del boom tecnológico ha sido la manera como las empresas han aprovechado la transición digital como una oportunidad para afrontar conjuntamente las incertidumbres que el propio cambio tecnológico plantea con sus continuas innovaciones. La circunstancia de crisis ha aumentado aún más las dudas generales y ha contado con el aval del Estado para tratar de implantar algunas acciones concretas orientadas a reducir el riesgo de las inversiones e imponer una suerte de política directriz (correcta o no) para afrontar el futuro inmediato (Morales, 2011, p. 3-4)

\footnotetext{
${ }^{5}$ Informe CMT 2009 - Comisión del Mercado de las Telecomunicaciones.

${ }^{6}$ Boletín Oficial del Estado. Ley 32/ 2003, de 3 de diciembre, General de Telecomunicaciones. BOE, número 264, del 4 de noviembre de 2003.

${ }^{7}$ Boletín Oficial del Estado. Real Decreto 920/2006, de 28 de julio, por el que se aprueba el Reglamento general de prestación del servicio de difusión de radio y televisión por cable. BOE, número 210, del 2 de septiembre de 2006 .
} 
En definitiva, la Televisión Digital por Cable, es el resultado de la digitalización de la señal de televisión para transmitirla a través de redes de cable coaxial o analógico, de cable híbrido o de fibra óptica, bajo el estándar DVB-C ${ }^{8}$ (Digital Video Broadcasting Cable). Más allá de la ampliación de la oferta de canales que permite el cable digital, lo más novedoso es la posibilidad que existe de ofrecer un variado abanico de servicios que no eran posibles con el cable analógico, fundamentalmente en tres servicios:
a. Telefonía
b. Internet
c. Vídeo Bajo Demanda (VOD-Video on Demand)

Mientras que el VOD es un servicio "propio" desarrollado por la industria de la televisión de pago, los servicios de telefonía e Internet eran ajenos a esta sector, lo que ha permitido el uso de la fibra en el cable digital, es el aprovechamiento de su capacidad casi ilimitada para ofrecer más servicios a los abonados. A esta oferta de servicios se la conoce como:

I. Doble Play - Además del servicio de televisión, se ofrece el servicio de telefonía o Internet.

II. Triple Play - Además del servicio de televisión, se ofrece telefonía e Internet.

III. Actualmente, los operadores de cable digital están ofreciendo un nuevo servicio a sus abonados a través de la telefonía móvil. A esta oferta se la conoce como Cuádruple Play.

Son estas ofertas las que día a día permiten que los servicios de la Televisión de Pago por Cable multipliquen las posibilidades de entretenimiento y acceso a la información. Entre los principales operadores encontramos: Ono, Telecable, R, Euskaltel ${ }^{9}$.

\subsection{La Televisión Digital de pago por satélite en España}

En España se comienzan a utilizar los satélites en la década de los 80 como contribución para la televisión tradicional de algunos eventos internacionales, aunque se entendía el uso del satélite como una oportunidad de desarrollo industrial y tecnológico que permitiría la ampliación de las redes de comunicación, o la creación de las mismas. El 7 de abril de 1989, el Consejo de Ministros aprueba el programa Hispasat 92, y autoriza la constitución de la sociedad Hispasat S.A para la explotación de sistemas de comunicación por satélite.

Una vez instaurado el programa de Hispasat 92, el estándar empleado en España en la Televisión Digital Vía Satélite (al igual que otros países de la Unión Europea), el DVB-

\footnotetext{
8 El DVB (Digital Video Broadcasting) es un organismo encargado de crear y proponer los procedimientos de estandarización para la televisión digital compatible, está constituido por más de 270 instituciones y empresas de todo el mundo.

${ }^{9}$ Informe Anual 2009 CMT - Comisión del Mercado de las Telecomunicaciones.
} 
S (Digital Video Broadcasting - Satellite) ${ }^{10}$, que permite una multiplicidad de servicios interactivos. Las primeras experiencias de TV de pago vía satélite, primero Canal+ y luego Cotelsat (ambos analógicos), y a partir de la necesidad de contar con una regulación específica para el sector, se publica en 1995 la Ley 37/199511, de Telecomunicaciones por Satélite. En enero de 1997 comienza a operar la primera plataforma digital de TV de pago vía satélite: Canal Satélite Digital (CSD), y su origen era la Sociedad General Cablevisión, S.A. (Sogecable), creada en 1992 por el grupo Prisa y Telefónica.

Canal Satélite Digital, además de ofrecer un paquete de servicios de canales de televisión, ofrecía la posibilidad de acceder a servicios interactivos de canales y de emisiones radiales, con lo cual, el espectro de posibilidades se amplió considerablemente.

En septiembre de 1997 se lanza Vía Digital (VD), como la segunda plataforma de TV de pago vía satélite, a través de Hispasat. La propuesta de VD para competir con CSD se basaba en la siguiente estrategia:

a. Incluir los canales autonómicos

b. Ofrecer contenidos nacionales y de productores independientes.

c. Apostar por la comunidad hispanoamericana

d. Un precio más bajo que CSD en el PPV de fútbol

Con estas ofertas, la llamada convergencia de medios y la posibilidad de acceder a servicios interactivos, la Televisión Digital de pago por satélite, incrementó el abanico de servicios que hasta ese día se ofrecían en España.

\subsection{La Televisión Digital de pago por ADSL en España}

La Televisión Digital por ADSL, como es el caso también de la televisión digital a través del cable o del satélite, es el resultado de la digitalización de la señal de televisión para enviarla a través de la línea telefónica, siendo el ADSL (Asymmetric Digital Subscriber Line) o "linea de abonado digital asimétrica" la más conocida y utilizada. Estas tecnologías utilizan una frecuencia más alta para transmitir a través de la línea telefónica, datos a alta velocidad (Internet) sin que interfiera con la voz, es decir, a través de la tecnología ADSL - en la línea telefónica se establecen tres canales: el de la línea telefónica normal, el de recepción de datos y el de envío de datos- .

En España la tecnología ADSL se lanza en 1999 de la mano de Terra, propiedad de Telefónica; más tarde el resto de las compañías del sector lanzarán servicios similares (Retevisión, Wanadoo, Inicia, Arrakis, Ya.com). Actualmente, entre las compañías de

\footnotetext{
${ }^{10}$ DVB-S (Digital Video Broadcasting - Satellite): es un sistema que permite incrementar la capacidad de transmisión de datos y televisión digital a través de un satélite.

${ }^{11}$ Boletín Oficial del Estado. Ley 37/ 1995, de 13 de diciembre, de Telecomunicaciones por Satélite. BOE, número 297, del 12 de diciembre de 1995
} 
Televisión Digital de pago por ADSL en España, encontramos Imagenio, Orange, Jazztel ${ }^{12}$.

\subsection{Internet y banda ancha en España}

La digitalización de la Televisión en sus servicios de pago de Cable, Satélite y ADSL permitió una mayor oferta de contenidos audiovisuales que modificaron las maneras de acceder a la información a través de la banda ancha en Internet.

Las nuevas redes, cuyo ritmo de despliegue es sostenido, están preparadas para soportar comunicaciones de banda ancha. Su diseño ha sido concebido desde la convergencia tecnológica: se trata de redes de nueva generación dotadas de capacidades multiservicio (una sola red para todas las aplicaciones y que soporta múltiples formas de acceso) (Fondevila, J. 2009: s/p)

Esto debido a que para visionar y/o descargarse películas, series de televisión u otros contenidos, cuanto más rápida es la conexión, menos tiempo se tarda en descargarse el contenido al ordenador u otro soporte. También es necesaria una conexión rápida, si se quiere utilizar el "streaming" en vez de la descarga. El "streaming" es una modalidad por la cual el contenido audiovisual no se descarga, sino que el usuario lo va viendo a medida que se va a emitiendo y no lo graba en su ordenador. La diferencia con la descarga es que no hay que esperar el tiempo de "descarga" del contenido al ordenador ( $u$ otro soporte) para visionarlo. Sin embargo, si se utiliza el "streaming", no queda una copia del archivo audiovisual para poder verlo en otro momento y/o copiarlo a un DVD u otro soporte. En cualquiera de los dos casos, cuanto mayor es el ancho de banda (conexión más rápida), menor es el tiempo de descarga y mayor es la calidad del "streaming".

Las formas tradicionales de relacionarnos con los medios, aparatos y contenidos han sufrido también transformaciones inducidas por la revolución tecnológica. La variedad de opciones y calidades para visionar y registrar señales audiovisuales de la televisión vía satélite y los receptores digitales, ha incrementado nuestra destreza instrumental, convirtiéndonos en espectadores expertos «proactivos» para seleccionar interactivamente la información de un inmenso repositorio que va creciendo y alimentándose diariamente de diferentes fuentes: desde materiales generados por las grandes productoras y distribuidoras hasta de jóvenes novatos en la creación audiovisual (Morales, 2011, s/p)

Con este nuevo panorama de acceso a la información la cifra de usuarios a Internet en España llega a 29.093.984 (diciembre 2009), con una tasa de penetración del 62,6\%. El crecimiento de usuarios a Internet entre el año 2000 y el año 2010 ha sido del 440\%. En relación con la Banda Ancha, en España hay (diciembre 2009) 9.786.578 conexiones de este tipo ${ }^{13}$. En cuanto a la evolución de las conexiones de banda ancha en

\footnotetext{
12 Informe Anual 2009 CMT - Comisión del Mercado de las Telecomunicaciones.

${ }^{13}$ Elaboración propia a partir de los datos de Internet World Stats y OCDE.
} 
España se observa un crecimiento de 188\% entre los años 2004 a 2009. Mientras en el 2004 el 82\% de las conexiones eran de menos de 1Mbps, y prácticamente solamente había 15.964 conexiones de entre 4Mbps y 10Mbps, en el año 2009 las conexiones de menos de $1 \mathrm{Mbps}$ no llegan al 1\% del total de conexiones, y las de entre $4 \mathrm{Mbps}$ y $10 \mathrm{Mbps}$ representan el $47 \%$. Estos indicadores nos ilustran claramente la velocidad con la que crece la penetración de la banda ancha en España, y que está relacionada con la transformación que ha vivido la industria de pago de televisión y su formato actual: el vídeo online.

\subsection{Consumo de Contenidos Digitales en España}

Según el Informe Anual de los Contenidos Digitales en España 200914, en España aumenta año tras año el consumo de contenidos digitales:

- La población española que consume Contenidos Digitales ha aumentado en 5,9 puntos porcentuales respecto a 2008 consumiendo actualmente el 70,3\% de la población algún tipo de contenido digital.

- En 2003 la mitad de las conexiones a Internet eran de banda estrecha; en la actualidad, el 97\% de los accesos a Internet ya son de banda ancha. La cobertura de las redes de banda ancha en España alcanza el 99\% de la población, una de las más elevadas del mundo.

- En el año 2009 las películas y las series se han convertido en el contenido digital más consumido por parte de los usuarios, siendo el 53,6\% de los españoles, 6,8 puntos más respecto a la medición anterior de 2008. El 49,4\% de los españoles tienen como uso más frecuente el visionado a través de la TDT y a través de medios on-line en tiempo real (streaming).

También se desprende de este estudio que el nivel de consumo de vídeo online en España, es mayor que el de la media Europea. En España el 87\% de los internautas ven vídeos en su ordenador, lo que nos sitúa por delante de Francia, Alemania, Italia y el Reino Unido. Por otro lado, y según el estudio Mediascope Europe $2010^{15}$ realizado por la Asociación Europea de Publicidad Interactiva EIAA (European Interactive Advertising Association), en febrero 2010, los españoles ocupan la quinta posición en el ranking de consumo de Internet de la Unión Europea, con 13,6 horas por semana, y con un crecimiento del 12,4\% en relación con el 2008 (12,1 horas).

\subsection{Televisión IP y Vídeo Online}

Con el crecimiento del visionado de Vídeo Online a través de Internet se hace necesaria la definición de la Televisión IP, a través de la Unión Internacional de Telecomunicaciones ITU (International Telecommunications Union).

El conjunto de servicios multimedia (televisión, vídeo, audio, texto, gráficos $\mathrm{y}$ datos) que son distribuidos por una red IP, los cuales deben poseer un nivel de

\footnotetext{
14 Ministerio de Industria, Turismo y Comercio.

15 Asociación Europea de Publicidad Interactiva: Estudio Mediascope Europe 2010.
} 
calidad de servicio, seguridad, interactividad y fiabilidad. También podemos referirnos a la Televisión IP utilizando la abreviatura TVIP o la abreviatura del término inglés IPTV. Se basa en el concepto de distribución de contenidos audiovisuales a través de Internet utilizando conexiones de banda ancha sobre protocolo IP, lo que permite desarrollar servicios y software para Televisión IP y WebTV que permiten publicar vídeo en "streaming" a través de Internet. Las soluciones de IPTV permiten poner en marcha proyectos de vídeo en Internet, Televisiones IP y WebTV accesibles a través de un navegador, donde las posibilidades de la televisión por Internet son ilimitadas: desde una infinidad de canales de televisión y música, e-learning, programación de televisión bajo demanda (VOD), streaming de video, live TV, publicidad dedicada, comunicación corporativa, entre muchos. La Televisión IP permite ofrecer una programación individualizada para cada usuario, así como nuevas formas de publicidad más interactivas, con un mayor control sobre la repercusión e impacto de los mensajes publicitarios. ${ }^{16}$

El vídeo online, por lo tanto, tendría las siguientes características:

a. Contenidos audiovisuales de distintas duraciones y de distintos géneros (películas, series, documentales etc.) que se ofrecen desde páginas (sitios, portales, servicios etc.) de Internet a los usuarios, y a los cuales los usuarios acceden utilizando distintos medios de conexión y visualizan a través de distintos soportes tecnológicos.

b. Los contenidos audiovisuales se consumen en modalidad a la carta, es decir, vídeo bajo demanda VOD (video on demand).

Además de las características existen distintos modalidades de vídeos online, pero a modo de simplificación y acotación, se clasificarán en 4 categorías:

a) Vídeos online para ver sin descargar: se conoce como "streaming".

b) Vídeos online para descargar: se conoce como "descarga".

c) Vídeos online con publicidad: se suele colocar al principio del vídeo.

d) Vídeos online por los cuales hay que pagar para visionar o descargar.

En cuanto a los dispositivos utilizados para el consumo del vídeo online se encuentran:

a) Ordenadores de sobremesa, portátiles y tablets PC's.

b) Teléfonos móviles, smartphones y PDA's.

c) Televisores tradicionales y los conectados a Internet (Connected TV's).

Es perceptible la combinación armoniosa de una infraestructura tecnológica consistente con contenidos atractivos. Así, la misión reside en ofrecer al espectador contenidos de calidad muy elevada. Para ello hay que utilizar redes de banda ancha y accesos de muy alta velocidad y, a poder ser, garantizados (Fondevila, J. 2009: s/p)

16 International Telecommunications Union: (www.itu.int). 
Las características, las modalidades y dispositivos del vídeo online permiten apreciar que este nuevo formato de creación audiovisual está ganando terreno en cuanto a consumo se refiere, debido a las múltiples posibilidades de visionado y las múltiples formas narrativas que se cuentan en ellos. Son los vídeos online una alternativa y una oportunidad de negocio para las plataformas de televisión de pago que existen en España, plataformas que están perdiendo día a día abonados y que a su vez, pueden tener en el vídeo online una oportunidad de negocio.

\subsection{Análisis e interpretación de los datos}

De acuerdo a la metodología planteada se presentan a continuación tres flujogramas que describen y analizan las respuestas de los profesionales entrevistados sobre la historia y transformación de la Televisión de Pago en España hasta llegar al auge del Vídeo on line. Las respuestas están relacionadas con las unidades temáticas seleccionadas para la metodología de este estudio.

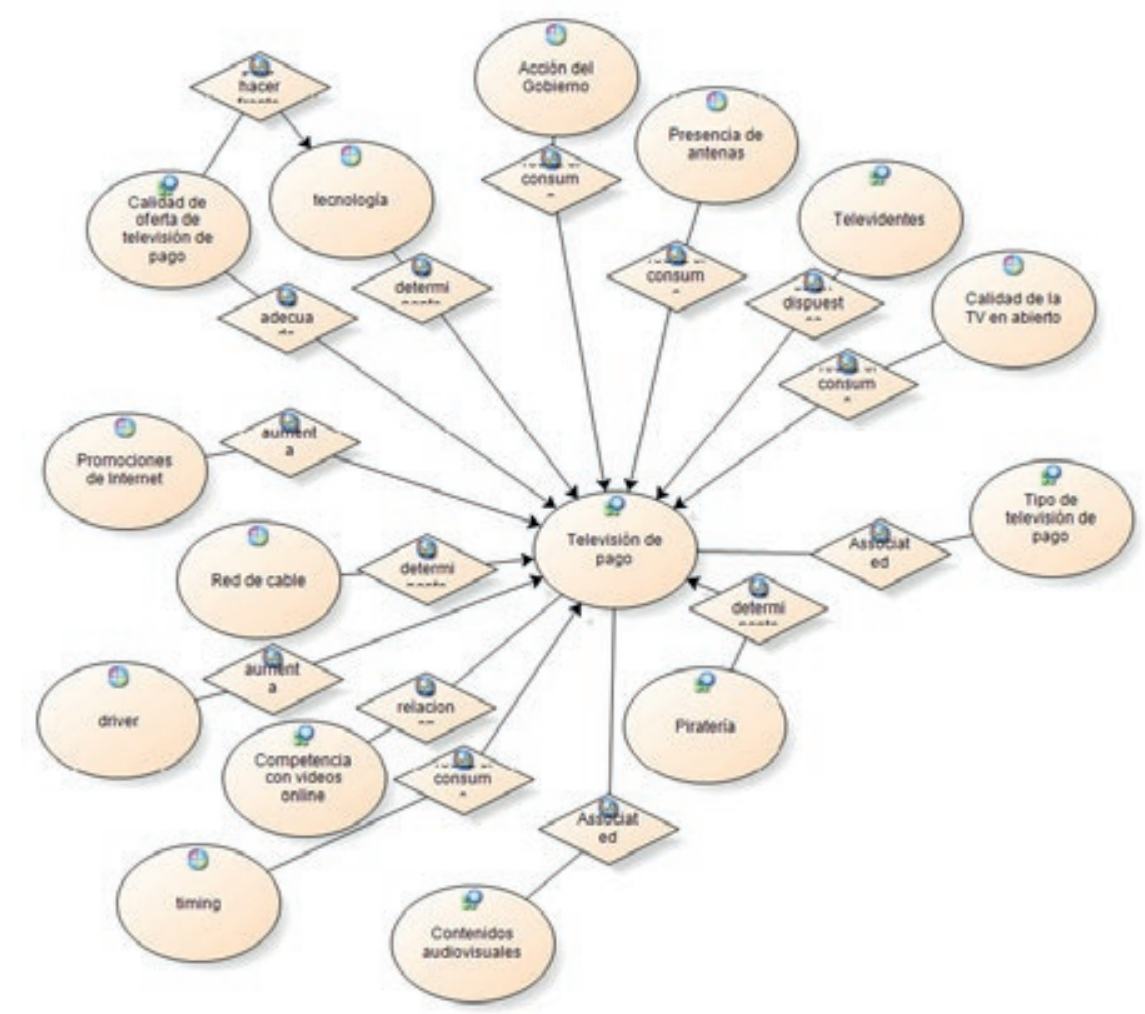

Figura 1. Flujograma Televisión de Pago en España

Fuente: Elaboración propia.

La expansión de la Televisión de pago en España, a partir de la década de los noventa, fue gestionada por empresas que poco o nada tenían que ver con el sector audiovisual o de telecomunicaciones, a excepción de Cableuropa/Ono, lo que se tradujo, según la mayoría de los profesionales encuestados, en una continúa distorsión y retroceso en las políticas de crecimiento del sector. Este hecho sumado a la intromisión permanente del estado en la industria del cable, a través de una 
empresa pública, como fue en su momento Telefónica y su filial Telefónica Cable, acentuaron dicho retroceso en la industria.

Los entrevistados, argumentan que dicha intromisión, volvió a repetirse -esta vez en el ámbito de la transmisión satelital- con el lanzamiento de Vía Digital (VD) en 1997, nuevamente, de la mano de Telefónica, para competir con el recién estrenado servicio de Canal Satélite Digital (CSD), que ocasionó, finalmente, la absorción de VD por parte de CSD a finales de 2003, y que a su vez dio como resultado la creación de Digital +. Los profesionales manifiestan que, esta nueva intromisión del gobierno propició que el número de abonados a la Televisión de Pago en España decreciera en el período $2002-2004^{17}$ y pasaran a las plataformas satelitales; hecho que también lo confirma el Informe de la Comisión del Mercado de las Telecomunicaciones (CMT). A este suceso de la historia de la Televisión de Pago en España se le conoce como la "guerra de las plataformas". Este suceso ha sido recordado por la mayoría de los entrevistados.

El lanzamiento de "Quiero TV" a finales de 1999, como primera plataforma de pago de Televisión Digital Terrestre, se recuerda, en palabras de los profesionales encuestados, como una inversión de pésima gestión, que produjo otro retroceso en el sistema de Televisión de Pago en España. Dos hechos se recuerdan de dicho proceso de gestión económica.

Por una parte, "Quiero TV" utilizaba una tecnología novísima que aún estaba sin explotar, y por otro lado, al implantar una tecnología novísima, desconocida por la teleaudiencia y los propios inversores del negocio, la empresa quería competir con las plataformas satelitales y con los sistemas de cable que empezaban a posicionarse en el mercado. La consecuencia fue que "Quiero TV" quebró en el año 2002 habiendo alcanzado solamente 120.000 abonados con una inversión de 900 millones de euros. La fallida experiencia de "Quiero TV" ocasionó que los recursos económicos de Retevisión (Auna) no se hubieran invertido en expandir sus sistemas de cable, al mismo tiempo que introdujo aún más competencia. Innecesaria, según los encuestados, pues el nuevo sistema de esta plataforma competiría con el servicio satelital de VD y CSD.

Otro factor que ha incidido en el crecimiento de la Televisión de Pago en España, en palabras de los profesionales, es el papel que juega la alta calidad percibida de la televisión abierta, según los televidentes españoles. La oferta de los canales generalistas públicos, los generalistas privados, las cadenas autonómicas y algunos locales, que se emiten gratuitamente, hacen que el mercado de la Televisión de Pago en España, tenga que pensar en estrategias de contenidos que llamen demasiada la atención de los televidentes. También, mencionan los entrevistados, que el nuevo sistema de Televisión Digital Terrestre que se implantó en 2010 hace que la competencia sea aún mayor, por la oferta gratuita de contenidos, y por ende las estrategias de captación de nuevos socios para la Televisión de Pago en España sean

17 CMT Informe 2009 - Comisión del Mercado de Telecomunicaciones. 
todavía más exigentes.

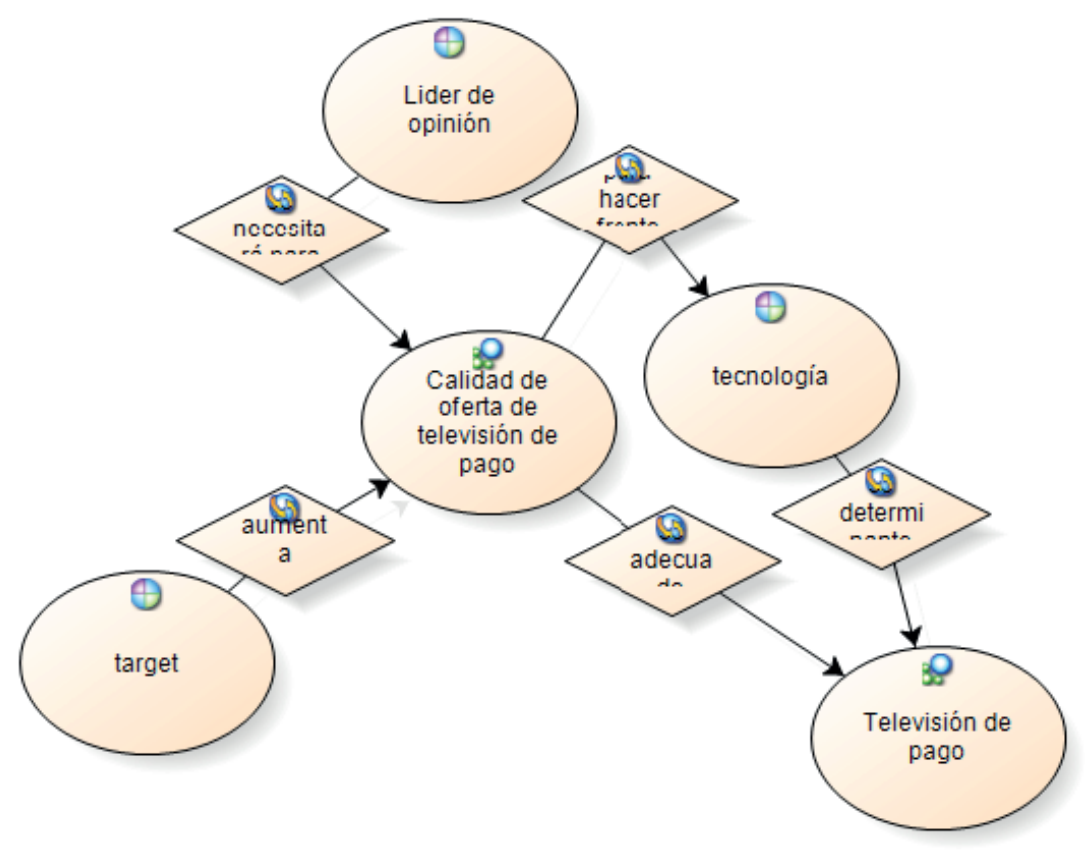

Figura 2. Flujograma calidad de la oferta de Televisión de Pago en España Fuente: Elaboración propia.

La televisión en abierto en España ofrece de forma gratuita dos contenidos, que han sido el reclamo, sobre el cual se ha construido inicialmente la industria de la TV de pago: el fútbol y el cine. Estos dos contenidos (aparte de los contenidos eróticos) fueron la base sobre la que lanzó Canal+ como el primer canal de Televisión de Pago hertziano en 1990, y que en 1997, pasó a ser satelital, convirtiéndose en el buque insignia de la plataforma satelital: el llamado Canal Satélite Digital.

Desde el lanzamiento de Canal+, el cine y el fútbol han ido incrementando su presencia en la televisión abierta de forma continuada. Actualmente, sobre todo el cine es parte integral de todos los canales en abierto. En cuanto al fútbol, y otras disciplinas, además de eventos deportivos, se ha ido incrementando su presencia en las televisiones públicas nacionales y autonómicas, como también en las privadas. Este incremento del fútbol y del deporte en general en la televisión abierta, ha llegado a su punto álgido a partir de lo que se conoce actualmente como la "guerra del fútbol", la que enfrenta, según los entrevistados, a los dos grupos de comunicación que están detrás de los nuevos canales analógicos generalistas privados lanzados en 2005: Cuatro (Sogecable) y La Sexta (Mediapro). Este enfrentamiento ha derivado en un incremento de programación en abierto en ambos canales de acontecimientos deportivos y fútbol, que ha perjudicado a la industria de Televisión de Pago en España, debido a que el fútbol era una de sus apuestas estrellas.

En cuanto a otros contenidos presentes en la televisión de pago, como son los documentales, los programas infantiles, las noticias, las series, etc. La televisión en 
abierto no se ha quedado atrás; su oferta ha ido incrementándose a lo largo de los últimos años, y actualmente se ha visto complementada y completada con el lanzamiento de los denominados canales "temáticos" en TDT, los cuales cubren los distintos géneros de forma especializada. Entre estos canales encontramos: Clan TVE (infantil), Sony, Antena Nova y Antena Neox (series y cine), Teledeporte (deportes), CNN+ e Intereconomía (noticias), MTV (musical), etc.

Dado que la industria de la TV de pago en España se ha desarrollado tarde, como mencionan los entrevistados, y debido a los factores que antes se han mencionado, la industria, actualmente, no cuenta con una masa crítica lo suficientemente grande (4 millones de hogares abonados - $25 \%$ de penetración) ${ }^{18}$ para ofrecer una mayor cantidad de canales de pago (en promedio 80 canales temáticos de pago), en comparación con mercados más desarrollados como el de Estados Unidos (500 canales) o el Reino Unido (500 canales). En este punto la oferta de la televisión abierta en España se contrapone a la oferta de la TV de pago. Su reducido número de canales en comparación con otros mercados, sumado a una creciente oferta de canales en abierto hacen que se la considere un servicio "prescindible", pero que nada tiene que ver realmente con su calidad o con su precio, sino más bien con el valor que la misma aporta en relación con la televisión abierta.

Volviendo a los contenidos de la televisión abierta vs los de la TV de pago, también se ha constatado a través de las entrevistas que muchos de los mejores contenidos que ofrece la televisión de pago, pasan antes o después por la televisión abierta, que quita exclusividad a la TV de pago por no contar con un Target de audiencia claramente definido y diferencial que le permita un crecimiento sostenido en un mercado audiovisual altamente competitivo y fragmentado como es el español. Sin embargo, desde la óptica pura de la calidad de los contenidos de la TV de pago, los mismos se adecuan perfectamente a su costo, y las plataformas de TV de pago cada vez incorporan más canales, mas servicios y más funcionalidades a su oferta como son: el Pago por Visión (PPV), Video Bajo Demanda (VOD), Videograbadores Personales Digitales (PVR), canales en Alta Definición, y más recientemente en 3D. Al mismo tiempo realizan constantes ofertas y promociones tendientes a captar nuevos clientes. En estos últimos años se ha ido incrementando la cantidad y la calidad de los servicios de las plataformas de pago, al mismo tiempo que su precio se ha ido reduciendo, convirtiéndola en una industria dinámica y en constante evolución.

${ }^{18}$ CMT Informe 2009 - Comisión del Mercado de Telecomunicaciones. 


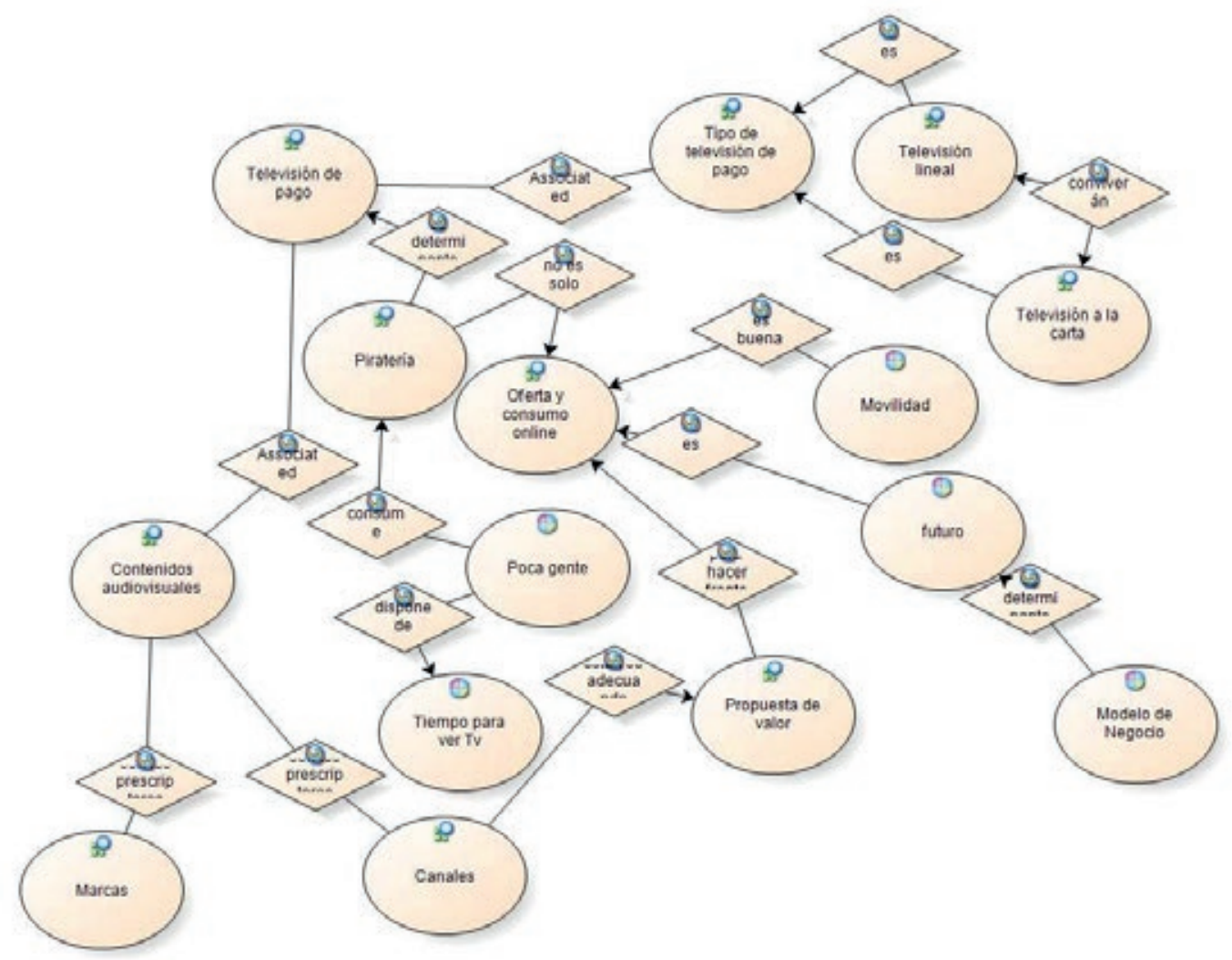

Figura 3. Flujograma de la oferta y consumo de Vídeos Online Fuente: Elaboración propia.

Los entrevistados coinciden en que la Industria de Televisión de Pago en España presenta incertidumbres en su futuro más inmediato, con respecto al auge y el aumento de consumo de vídeos online. Cada profesional, desde su particular experiencia profesional y de acuerdo a los puestos que ocuparon y ocupan (cargos relacionados con la Televisión e Internet) tienen lecturas diferentes, con respecto al nuevo fenómeno del aumento del vídeo online. Sin embargo, todos coincidieron en el hecho de que las nuevas generaciones de "nativos digitales" tienen unos hábitos de consumo distintos a los de su generación (todos los profesionales entrevistados tienen más de 40 años), que permiten que el impacto que tienen los vídeos online se encuentre en aumento. Los profesionales coinciden, además, que este aumento del vídeo online se puede convertir en una oportunidad de negocio para la Industria de Televisión de Pago en España. Argumentan que los hábitos de consumo se han transformado y que dichos hábitos deben ser escuchados, estudiados y a su vez representados en las nuevas ofertas de esta Industria de Televisión.

Otros puntos en los que coincidieron los entrevistados fueron:

a. El concepto de contenidos cuando se quiera, desde donde se quiera y como se quiera despiertan en los consumidores mayores oportunidades de visionado debido a que cada vez existen más dispositivos conectados a través de los cuales se puede acceder a este tipo audiovisuales (televisores "conectados", ordenadores y tablets PC's, teléfonos móviles, consolas, set top boxes, PVR's, y 
reproductores de DVD “conectados").

b. El protocolo IP como lenguaje universal de conexión y consumo de contenidos permite que esta tecnología de transmisión esté estandarizando la oferta y el consumo de contenidos audiovisuales, que a su vez se traduce en otra oportunidad de negocio.

Con respecto al futuro de la Televisión de Pago en España los entrevistados opinan:

a. Los dos entrevistados que pertenecen a la industria de la televisión reconocen que el vídeo online es un reto para la TV de pago. Opinan que la TV de pago no desaparecerá, sino que tendrá la capacidad de adaptarse a estos nuevos escenarios que presentan los nuevos consumidores.

b. Los entrevistados provenientes de la industria de Internet son más pesimistas en relación con el futuro de la industria de la TV de pago. Sostienen que la Industria de Internet es el nuevo paradigma y el nuevo Driver de consumo de contenidos audiovisuales.

Los dos grupos coinciden en que las marcas y los canales que producen o prescriben los contenidos, jugarán un papel determinante en el consumo de los mismos. Argumentan que las grandes marcas y los canales no desaparecerán sino que adaptarán sus ofertas a las nuevas tecnologías y a los nuevos dispositivos de transmisión, a su vez, que se adaptarán a los nuevos hábitos de los usuarios. Por lo tanto, se puede concluir que los entrevistados, indiferentemente, del sector al que pertenecen son conscientes que estas transformaciones, en ambos sectores, se constituyen en una nueva oportunidad de negocio. Para explotar el auge del vídeo online, todos coinciden en que los dos principales modelos son la publicidad y el transaccional (pago unitario y suscripción). Sin embargo el peso que tiene cada uno está por definirse. Se debe tener en cuenta, según los entrevistados, que la nueva industria del vídeo online está aún en sus inicios; consideran que desde el punto de vista de su explotación económica, se encuentran en una etapa embrionaria y en permanente evolución. A su vez que la Industria de Televisión de Pago, a pesar de no estar en una etapa embrionaria, también se encuentra en permanente transformación.

Estas dos transformaciones son las que se constituyen en oportunidades de negocio, pues ambas Industrias pueden coexistir en ofertas de consumo, siempre y cuando se mejore, ante todo, argumentan los entrevistados sus ofertas de contenidos, los cuales deben de presentarse como únicos y novedosos.

\section{CONCLUSIONES}

En los resultados obtenidos de las entrevistas, así como del estudio de material bibliográfico sobre la transformación y la evolución de la Televisión de Pago en España, se aprecia que las televisiones de pago están haciendo, constantemente, un esfuerzo para ajustar su oferta de valor a un mercado audiovisual cada día más 
competitivo y fragmentado.

La transformación y la evolución de la Televisión de pago en España se debe a tres factores primordiales: la tecnología, la legislación y la redefinición constante del sector de la industria audiovisual. Que si bien estos tres factores han permeado la totalidad de la transformación de este sector, actualmente, se añade la posibilidad que brinda la tecnología de Internet en banda ancha, y el vídeo online, para ser más específicos, como una amenaza a la industria. Sin embargo, esta amenaza, que también contiene los tres factores antes descritos, se puede convertir en una oportunidad de negocio, y en una nueva transformación y evolución de la televisión de pago. La tecnología constantemente se está transformando, la legislación española trata de aglutinar a este conglomerado empresarial, con unos deberes y derechos, a la vez, que el sector trata de ofrecer unos servicios para una audiencia cada día más exigente con lo que consume. Así el vídeo online, por su crecimiento en los últimos años, se constituye en un nuevo paradigma de consumo que se puede transformar en una oportunidad de negocio, en una oportunidad de negocio para la televisión de pago.

La industria de la TV de pago está reaccionando rápidamente, buscando adecuar su propuesta de valor para mantener su base de abonados, y al mismo tiempo crecer, con iniciativas como la de Televisión en Todas Partes (TV Everywhere). Este tipo de iniciativas con las que se está experimentando, tienen como objetivo ofrecer los canales, contenidos y servicios de las plataformas de pago a través de Internet, como respuesta al incremento que hacen sus abonados de la red para el consumo de contenidos audiovisuales. En este sentido, la industria de la TV de pago en España se está esforzando para adecuar su oferta a través del incremento de sus servicios (canales, contenidos y servicios interactivos), y la reducción del precio de los mismos, pero debe esforzarse aún más y debe experimentar -en la medida de sus posibilidades, con nuevos servicios orientados a los internautas como TV Everywhere, que le permitan volver a crecer.

\section{REFERENCIAS}

BOE (2006). Real Decreto 920/2006, de 28 de julio, por el que se aprueba el Reglamento general de prestación del servicio de difusión de radio y televisión por cable. BOE, (210), del 2 de septiembre de 2006

BOE (1998). Ley 11/1998, de 24 de abril, General de las Telecomunicaciones. BOE, (99), 25 de abril de 1998.

BOE (2003). Ley 32/ 2003, de 3 de diciembre, General de Telecomunicaciones. BOE, (264), del 4 de noviembre de 2003

BOE (1995). Ley 37/ 1995, de 13 de diciembre, de Telecomunicaciones por Satélite. BOE, (297), del 12 de diciembre de 1995. 
BOE (1995). Ley 42/ 1995, de 22 de diciembre, de las Telecomunicaciones por cable. BOE, número 0306, 23 de diciembre de 1995.

Estudio Mediascope Europe 2010 (2010). Asociación Europea de Publicidad Interactiva.

Fondevila- Gascón, J. F. (2009). La Televisión IP (IPTV) y la transmisión mediante VDSL: realidad y perspectivas de negocio, en Vivat Academia (115). Consultado el 2 de Marzo del 2011, Disponible en: www.ucm.es/info/vivataca/numeros/n105/articulo.htm\#TV

Informe CMT 2009 (2010). Comisión del Mercado de las Telecomunicaciones.

International Telecommunications Union (2010). Consultado el 2 de Marzo del 2011, Disponible en: $\underline{w w w . i t u . i n t}$

Krippendorff, K. (1990). Metodología de Análisis de contenido: Teoría y práctica, Barcelona: Paidós Comunicación.

Morales-Morante, L. F. (2011). Entre la TDT y el ordenador: nuevas tendencias tecnológicas, empresariales y de consumo alrededor de la ficción audiovisual en España, en Vivat Academia. (114). Consultado el 2 de Marzo del 2011, Disponible en: www.ucm.es/info/vivataca/numeros/n114/DATOSS.htm

\section{María Luisa García Guardia}

Profesora Contratada Doctora en la Facultad de Ciencias de la Información en la Universidad Complutense de Madrid. Asesora de proyectos sobre Publicidad, TICs, Sostenibilidad y Educación .Miembro de los grupos de investigación Socmedia y Museum I+D+C.

\section{Oscar Estupiñán Estupiñán}

Doctor en Ciencias de la Información. Universidad Complutense de Madrid. Máster en Comunicación y Producción Audiovisual. Universidad Internacional de Andalucía. Miembro de la revista Icono14. 